\title{
ALLERGY TO ENDOGENOUS HORMONES AS A CAUSE OF KERATITIS ROSACEA*†
}

BY

\author{
B. ZONDEK, J. LANDAU and Y. M. BROMBERG \\ From the Gynaecologic and the Ophthalmologic Departments of \\ the Rothschild Hadassah University Hospital, and the Hormone \\ Research Laboratory of the Hebrew University, Palestine.
}

ZONDEK and Bromberg' ${ }^{1}$ have found that patients suffering from typical allergic symptoms (asthma, rhinitis vasomotoria, urticaria, angioneurotic oedema, etc.) and certain other complaints not usually classified as such (premenstrual tension, pruritus vulvae, migraine, etc.) show in a certain percentage of cases hypersensitivity to their own hormones (" endocrine allergy "). In an earlier paper observations on women with symptoms depending on the genital cycle and related to menstruation or menopause were reported. It was suggested, however, that hypersensitivity to endogenous hormones can also occur in men and women quite independently of genital function.

The condition of endocrine allergy can be detected by an active intracutaneous test. The reaction to a steroid hormone is considered positive when 24-48 hours after intracutaneous injection of $0.1 \mathrm{mg}$. of steroid hormone dissolved in $0 \cdot 1$ c.c. of specially purified olive oil, a red or pink, slightly elevated and frequently itching papule occurs at the site of the injection. Reactions to insulin $(0.1 \mathrm{c.cm} .=0.01$ units $)$ or gonadotropin $(0.1 \mathrm{c.cm} .=0.1$ units $)$ are read as positive when 1-2 hours after injection of the hormone in physiological saline solution a wide erythema with urticarial swelling occurs at the site of the injection. The characteristic local reaction is accompanied in certain cases by a general reaction (angioneurotic oedema, urticaria, rhinitis vasomotoria, etc.) which affords further confirmation of the diagnosis.

Presence of specific allergy antibodies (reagins) in cases of 'endocrine allergy has also been demonstrated by the passive transfer test according to Praussnitz and Kuestner, and by spontaneous endogenous passive transfer tests..$^{1,2}$

The cases selected for the original series of examinations were all of unknown aetiology from a group which had been thoroughly investigated, and in which a condition refractory to treatment had persisted for a considerable period (more than 2 years). In several of the cases desensitisation treatment was applied. Good results were obtained by this means in a large percentage of the group. Details of the treatment and the technique applied have been presented in earlier papers. ${ }^{1,2}$

* This investigation was aided by a grant from the Ella Sachs-Plotz Foundation. t Received for publication, December 20, 1946. 
Certain eye diseases of still unknown aetiology (like a case of undetermined keratitis superficialis) were also investigated in the course of this study in endocrine allergy, and good therapeutic results were obtained by desensitisation treatment with allergenic hormones. ${ }^{1,2}$

The numerous theories of the pathogenesis of keratitis rosacea seem to point to the lack of any definite knowledge as to its true aetiology. It seemed to us that allergy to endogenous hormones might account for a number of these cases.

Keratitis rosacea is characterised by garland-like dilatation of the limbal vessel, vascular invasion of the cornea, and appearance of sub-epithelial opacities in the anterior corneal layers. The opacities gradually increase and finally are apt to cause impairment of vision. The subjective symptoms include photophobia, burning, foreign body sensation, mild pain, and general malaise which disables the patient. Other ocular disturbances, such as blepharitis and conjunctivitis, and rarely keratitis rosacea (and iritis rosacea) are also observed. The syndrome is well-known to occur with or without facial involvement. Rosacea faciei is characterised by appearance on the nose and cheeks of vascular dilatations, initially transient and later permanent, which may lead to a persistent telangiectasia.

The disease is of long duration, progredient in character, and a satisfactory therapy has not yet been devised.

Many theories (contradicting each other), concerning the aetiology of rosacea have been advanced. Bacterial pathogenesis, ${ }^{3}$ disturbances in the alimentary tract (hypochlorhydria), ${ }^{4}$ and secretory and angiomotor neurosis of a hereditary character ${ }^{5}$ have been suggested. A common assumption that the cause of keratitis rosacea is dietary abuse, exhaustion and irregularity in taking meals has been shown to be erroneous. ${ }^{6}$

Recently it has been suggested that riboflavin deficiency is the cause of keratitis rosacea, and may lead at the limbus to vascular dilatation such as is observed in keratitis rosacea. It has been pointed out, however, that the corneal vascularisation due to ariboflavinosis differs from that of keratitis rosacea. ${ }^{7}$ 'The efficacy of riboflavin treatment in keratitis rosacea has been doubted. ${ }^{8-10}$

The obscurity of the aetiology of keratitis rosacea is matched by the uncertainty of its therapy. It is generally recognised that treatment of this condition must be general and constitutional. ${ }^{11}$ Local treatment (ichtho-zinc) is only palliative. General treatment has been attempted along different lines, $e . g$., treatment of possibly present gastric malfunction, protein shock therapy, elimination of septic foci, ${ }^{11}$ hormonal treatment with corticosterone, ${ }^{12}$ and recently riboflavin $6^{6}, 7,13$

Tests for presence of hormonal allergy have been performed in 
6 cáses of keratitis rosacea ( 3 men and 3 women; duration of disease from 4 to 20 years). All cases were refractory to accepted methods of treatment and showed gradual progress. The six cases of keratitis rosacea were tested with regard to several hormones with the following results: In all of them hypersensitivity to testosterone only was found (Fig. 1). Desensitisation treatment with small gradually increasing doses of the hormone produced in every case an improvement of the condition of the patient.

CASE 1.-G.A., female, aged 40 years, housemaid, regular menstrual periods, married for 16 years; three deliveries and one abortion. The father died of diabetes. Two children exhibited

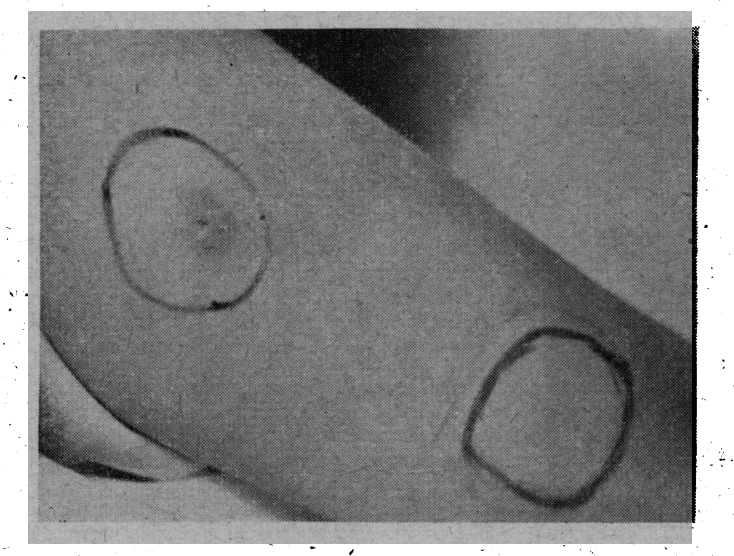

Fíg. 1.

Left : Positive intracutaneous test with testosterone in óly solution. Right: Negative test with oil control.

allergic manifestations. The first child, a 6 year old girl, is suffering from chronic urticaria of unknown aetiology. The second child, a 4 year old girl, suffers from spastic bronchitis. No other peculiarities were found in the patient's history. Rosacea faciei, with symptoms so severe that they were ascribed to lupus miliaris, set in 12 years ago. Concomitantly eye disturbances-blepharoconjunctivitis and keratitis rosacea-developed. The disease gradually became aggravated in the following years, but remissions occurred from time to time. The patient suffered very much from photophobia, burning and lacrimation which disabled her to a great extent. It is noteworthy that all these complaints subsided during pregnancy, but recurred in the period of lactation. There were no other symptoms that could in any way be related to the genital cycle.

Present condition. Extensive rosacea of the cheeks and nose and enlargement of the vessels, were seen giving the face a dark red 
aspect; furthermore, bilateral blepharo-conjunctivitis was present, the vessels of the bulbar conjunctiva were markedly enlarged on both sides. The vessels around the limbus presented a garlandlike appearance and partly encroached upon the cornea. Subepithelial infiltrates, some of them reaching the borders of the pupils, were observed, penetrating partly into the outer layers of the corneal parenchyma (Fig. 2). General examination revealed normal findings : Blood chemistry normal ; urine normal; Wassermann reaction in blood negative; blood picture: erythrocytes

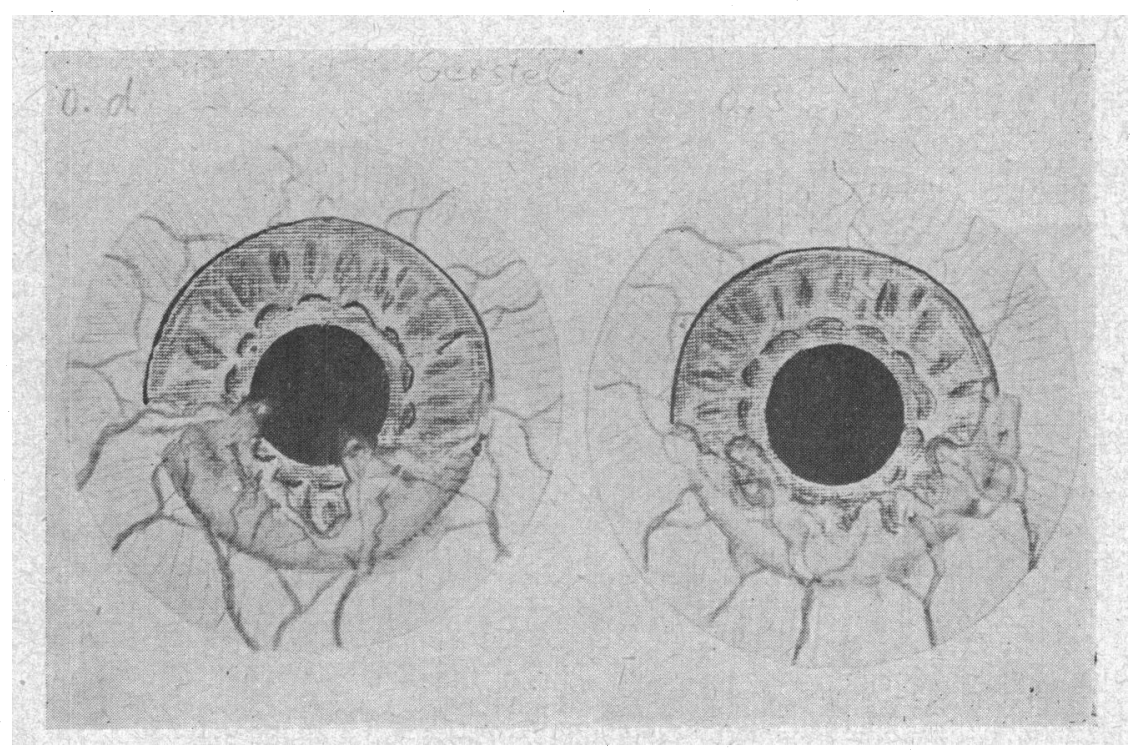

FIG. 2.

Left and right eye : patient (Case No. 1) suffering from keratitis rosacea before treatment. Note garland-like enlargement of the vessels around the limbus corneae.

$4,840,000$, slight leucocytosis-11,500, eosinophiles 3 per cent., blood sedimentation normal. The following intracutaneous tests for presence of hormonal allergy were carried out :-

(1) ovarian series : oestradiol, oestrone, progesterone, pregnandiol ;

(2) adrenal series : corticosterone, testosterone, androsterone;

(3) control series : cholesterol and oil.

The tests showed clearly a strong hypersensitivity to testosterone. Control tests with oil alone and cholesterol were negative. The desensitisation treatment consisted of subcutaneous injections of gradually increased doses of testosterone dissolved in oil (20 successive daily injections of doses ranging from 0.01 to $1 \mathrm{mg}$.). This therapy produced a definite improvement. The subjective 
symptoms of the patient improved, the photophobia disappeared, there was no burning sensation, and the limbal vascular dilatation was considerably diminished. Four weeks after release from hospital, the patient was still free from her former symptoms, and only four weeks later she came to see us with a mild recurrence of the inflammatory symptoms. The corneal infiltrations extended from the nasal limbus to the borders of the pupils and showed characteristic degenerative changes. The patient was again given 10 injections of testosterone $(0.1$ to $1.0 \mathrm{mg}$.) and for consolidation of the treatment a tablet of $10 \mathrm{mg}$. was implanted subcutaneously.

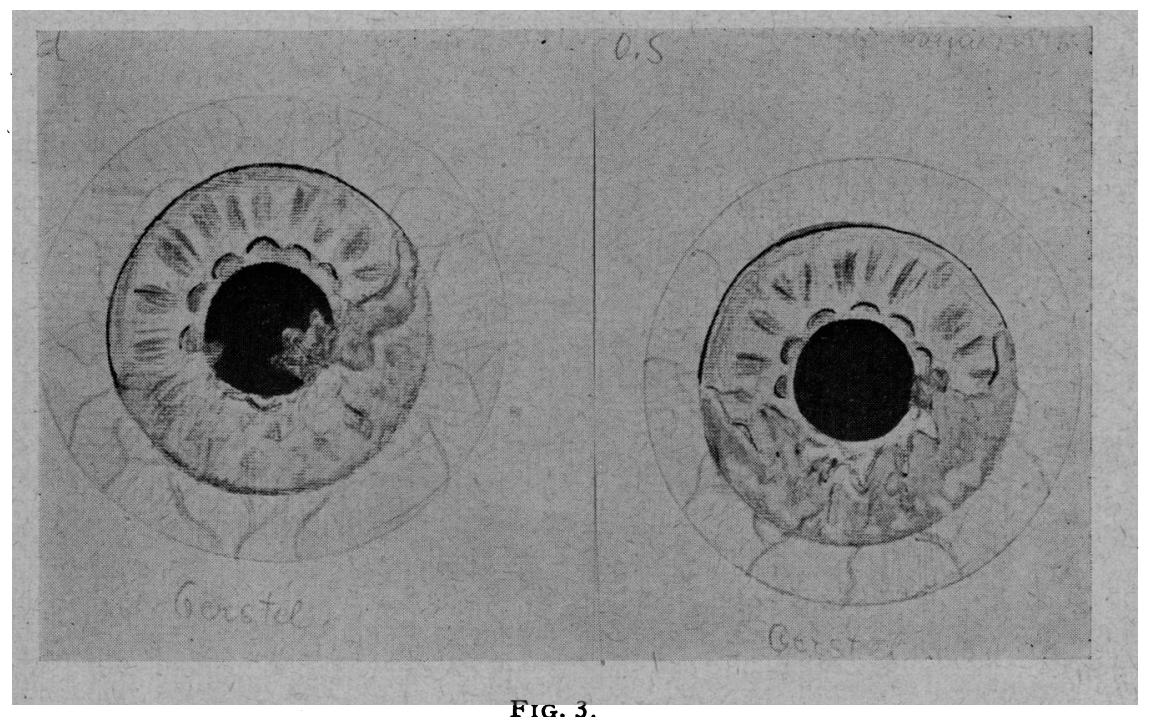

Same patient after desensitisation with testosterone. Note recession of the vascular engorgement.

This time the patient remained free from symptoms for a period of 3 months. So long an interval had not previously been experienced by the patient except during pregnancy. Then a mild inflammation set in. $25 \mathrm{mg}$. of testosterone were again implanted. Ten days after implantation an acute inflammatory reaction was present in both eyes which lasted for two weeks and was ascribed to a possibly excessive dose. Apart from this reaction the patient remained free from complaints. There was considerable improvement of the rosacea faciei. Vascular dilatation in the nose and cheeks regressed markedly, and the face became decidedly less red. Vascular injection of the conjunctiva bulbi and at the limbus diminished. The corneal opacities were unchanged after an observation period of one year (Fig. 3). Local therapy had been avoided as far as possible during this interval. 
CASE 2.-K.W., male, aged 45 years, a policeman by occupation. Suffered for many years from chronic dyspepsia possibly as a sequel to chronic amoebiasis. For 6 years rosacea faciei with involvement of the eyes-conjunctivitis and keratitis rosacea. Two years before, the condition of the right eye became so serious that the patient had to be admitted to the hospital. Recurrent ulcers at the temporal border of the cornea were present at that time. Local treatment (cauterisation) and general riboflavin and vitamin A therapy were given: The recurrent inflammation of the eyes greatly hampered the patient at his work.

Present condition. Moderately severe rosacea faciei, bilateral blepharo-conjunctivitis, rosacea, particularly manifest in the left

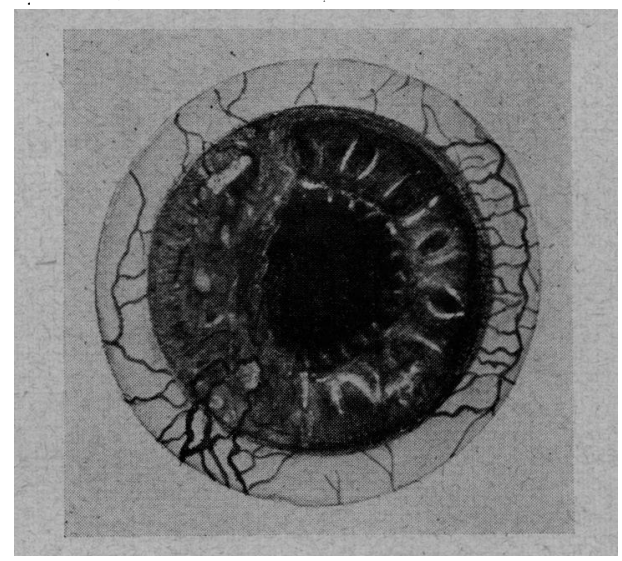

- Fig. 4.

Left eye of patient No. 2 with typical keratitis rosacea before treatment. Note strong pericorneal injection of vessels protruding into the cornea.

eye as characteristic vascular dilatations, partly forming a garland over the cornea; the superficial corneal layers were infiltrated (Fig. 4). Wassermann reaction in the blood was negative.

Intracutaneous test with hormonal allergens revealed a positive reaction only to testosterone. The tests were carried out with $0 \cdot 1$ $\mathrm{mg}$. and smaller amounts of testosterone, a positive reaction being obtained even with $0.001 \mathrm{mg}$. of hormone.

Desensitisation treatment, consisting of a daily subcutaneous injection of a gradually increasing dose of testosterone, ranging from $0.001 \mathrm{mg}$. to $1.0 \mathrm{mg}$., was given for one month. The condition of the patient improved and the symptoms disappeared. After two months, mild recurrence of the symptoms was experienced. Ten more injections of testosterone in doses ranging from $0.1 \mathrm{mg}$. to $1.0 \mathrm{mg}$. were given, the treatment being consolidated by the 
implantation of a pellet of $10 \mathrm{mg}$. of testosterone. Three months later a second pellet of $10 \mathrm{mg}$. was implanted. During the period of treatment only transient and mild manifestations lasting 2-3 days were observed. Eventually, a third pellet of $10 \mathrm{mg}$. was implanted. Local therapy was not employed during the whole time. The condition of the patient improved definitely, as there were no subjective symptoms and the vascular injection receded almost completely (Fig. 5). The rosacea faciei definitely improved as well. The patient, unhampered by any ailment, is again at work. The period of observation was twelve months.

CASE 3.-B.Z., female, aged 62 years, suffered for 20 years from keratitis rosacea accompanied by vascular dilatation on cheeks

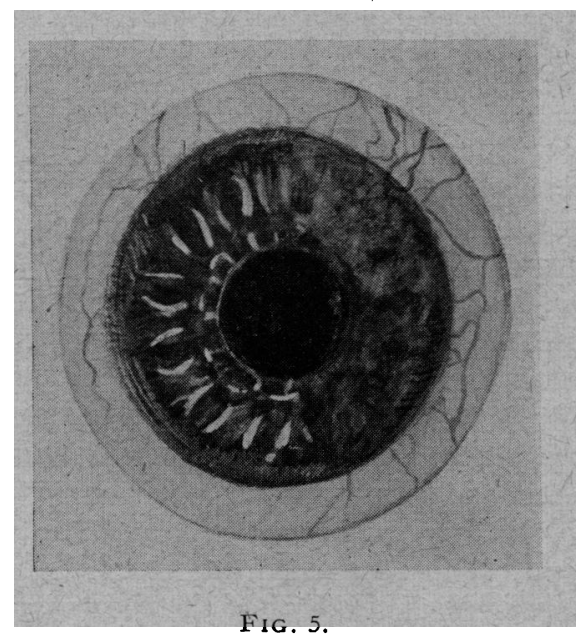

Left eye of same patient after desensitization with testosterone. Note disappearance of the vascular injection.

and nose. Recurrences of keratitis rosacea were so frequent that the patient was seriously handicapped in her work. The vessels around the limbus invaded the cornea. Sub-epithelial infiltrates reaching to the pupil area, in both corneae were particularly advanced on the right side. The patient complained of an intolerable almost constant sensation of burning, photophobia and of lacrimation. Intracutaneous tests with several hormonal allergens revealed a strong, positive reaction only in respect to testosterone. Acute inflammatory reaction developed in both eyes during the performance of the tests. Desensitisation treatment with gradually increasing daily doses of testosterone (from $0.01 \mathrm{mg}$. to $1 \mathrm{mg}$.) in a series of 20 injections administered subcutaneously was undertaken and finally a $10 \mathrm{mg}$. pellet of testosterone was implanted. Since this treatment, all the subjective symptoms as well as the 
vascular injection receded almost completely. The vascular dilatation on cheeks and nose also greatly improved. During the three months following the conclusion of the treatment, the patient, for the first time in many years, was free from complaints.

The following three cases will be reported briefly as they have been under observation for a short period only ; in the first of them desensitisation treament has not yet been instituted.

CASE 4.-R.K., man, aged 42 years, mechanic by occupation, suffered for 5 years from rosacea faciei accompanied by keratitis rosacea. Failed to respond to different treatments, local and general, given during this period. Intracutaneous tests made with different steroid hormones gave a positive response only in respect to testosterone. For personal reasons, it was necessary, in this case to postpone the treatment.

CASE 5.-The patient, a woman, suffered from rosacea faciei with keratitis rosacea. The symptoms were of long standing and resisted all earlier attempts at therapy. The intracutaneous tests with steroid hormones revealed hypersensibility to testosterone only. Desensitisation treatment was instituted and produced a marked improvement. (A detailed report on this case will be published by Dr. Lass, Allergist, Hadassah Hospital, Tel-Aviv.)

CASE 6.-A.K., man, aged 65 years, a theological scholar, suffered for the past 17 years from keratitis and conjunctivitis rosacea with a mild facial involvement. Two months previously he, suffered from a severe exacerbation of his ocular condition which did not yield to any local treatment. The intracutaneous tests with hormones revealed hypersensitivity to testosterone only. The treatment with gradually increasing doses of testosterone produced a striking improvement at the end of the first week, after which all inflammatory signs receded and the patient was able to resume his work.

\section{Discussion}

Allergy is considered to be the cause of a variety of eye diseases. In general the disturbance is ascribed to hypersensitivity of the ocular tissues to exogenous proteins. It has been assumed that human eye tissue can be allergic also to endogenous products of metabolism. In sympathetic ophthalmia, for instance, hypersensitivity to endogenous uveal pigment has been claimed. ${ }^{14} \mathrm{~A}$ positive cutaneous reaction to intradermal injections of uveal pigment extract has been demonstrated in cases of this kind. 15,16 Histologically cutaneous changes in this allergy resembled the histological changes in the eye. ${ }^{17}$ Allergy to endogenous iens protein has also been considered to be a cause of post-operative or post-traumatic endophthalmitis. ${ }^{18,19}$ In our cases it seems necessary to assume the presence in the ocular tissue of a state of allergy 
to endogenous hormones. It is of interest that in these cases the endocrine allergic reaction was not directed to protein hormone substances but to steroid hormones of a relatively simple structure. It is remarkable that in the six cases (three men and three women) examined by us the responsible hormonal allergen was always testosterone. This hormone is produced not only by Leydig cells of the testis, but also by adrenal tissue, and this circumstance probably explains the occurrence of an allergy to this substance in mature women. Our findings, pointing in one direction, of course do not exclude the possibility that other aetiological factors may also play a rôle in keratitis rosacea.

Treatment of hypersensitivity to endogenous hormones consists of specific desensitisation by daily subcutaneous injections of the offending specific hormonal allergen, in small and gradually increasing doses and in the present cases with testosterone. All cases showed definite improvement under this therapy. However, mild and transient recurrences were observed after a long interval following the completion of the treatment course. Regular repeated implantations of pellets containing $10 \mathrm{mg}$. (or may be even less) of the allergen at intervals of 3-6 months are therefore indicated as a means of consolidating the therapy. The initial doses of testosterone employed in desensitisation should be very small, never exceeding $0.1 \mathrm{mg}$., and the increase should be gradual. These precautions seem to be necessary since focal reactions have been observed after administration of larger doses, as witnessed in our first case. Similar precautions should be observed in pellet implantations.

Therapeutic results in keratitis rosacea must, as a rule, be interpreted with great caution, as spontaneous remissions occur frequently. Nevertheless, the results described above lead us to the conclusion that the treatment was specific. The subjective symptoms improved and the objective signs, the limbal and corneal vascular dilatation, also changed for the better. All patients were relieved, symptom-free periods of equally long duration had not occurred in the absence of treatment or after other treatments. Recurrences were noted in the form of slight vascular injection which, however, receded promptly after a second course of desensitisation with testosterone. An effect on the permanent degenerative corneal changes could of course not be expected.

Endocrine allergy affords a possible explanation for the unknown aetiology of certain diseases. While in some of them the offending hormonal allergen may vary (e.g., in urticaria positive reactions were found with regard to oestrone, testosterone and corticosterone) it is noteworthy that in our small group of cases of keratitis rosacea all showed allergy to one and the same hormone,

Proceeding from the assumption that the ocular tissues are 
capable of allergic reaction to endogenous hormones, other eye diseases of obscure aetiology and long duration, and in which an allergic basis could be suspected, have been studied from this point of view (chronic, periodically recurrent conjunctivitis and undetermined cases of keratitis superficialis as well as other diseases ${ }^{2}$ ). The results obtained in these cases owing to desensitisation treatment with the respective hormonal allergens have been encouraging.

\section{Summary}

1. Six patients with keratitis rosacea associated with rosacea faciei ( 3 men and 3 women) of long duration which had proved refractory to the usual methods of treatment, were examined for hypersensitivity reactions to endogenous hormones.

2. In all cases allergy to testosterone was demonstrated by positive skin reactions to intracutaneously injected testosterone. Other hormones (oestrone, oestradiol, progesterone, pragnandiol, corticosterone, insulin, gonadotropin) gave negative reactions.

3. Desensitisation treatment by a course of subcutaneous injections of testosterone in gradually increased doses and implantation of pellets containing $10 \mathrm{mg}$. of testosterone propionate produced satisfying results.

4. Testosterone gave relief only when given in low, gradually increased doses; administration of large doses may cause a severe exacerbation.

5. The finding of allergy to endogenous testosterone in a small number of cases of keratitis rosacea does not exclude the possibility :-

(a) that other endogenous hormones may act as allergens in some cases ;

(b) that other causative factors are involved in the aetiology of keratitis rosacea.

The results achieved were as follows :-

(a) Complete and prompt cessation of the subjective symptoms, photophobia, lacrimation and sensation of burning in the eyes.

(b) Considerable improvement or disappearance of the limbal and corneal vascular dilatation, as well as a marked improvement of the facial rosacea.

(c) Mild recurrences in some instances which promptly improved after administration of a second course with testosterone treatment.

We are indebted to Prof. A. Feigenbaum, Head of the Ophthalmological Department, Rothschild Hadassah University Hospital, 
for his help and keen interest in this paper, and'to Dr. B. Mitterstein, First Assistant of the Ophthalmological Department. We are furthermore indebted to Dr. Hilde Rosenburger for the drawings in Figures 2-5.

\section{LITERATURE}

1. Zondek, B. and Bromberg, Y. M.-Jl. of Allergy, Vol. XVI, pp. 1-16, 1945.

2. In press.

3. Galloway and Govadly.-Proc. Roy. Soc. Med., Vol. XX, p. 408 ; cit.f. DUKE-ELDER, Text-book of Ophthalmology, Vol. II, p. 1721.

4. RYLE and BARBER.-Lancet, Vol. XI, p. 1195, 1920 ; cit.f. DUKE-ELDER, Text-book of Ophthalmology, Vol. II, p. 1721.

5. HallopeaU, H.-Handbuch der Haut- und Geschlechtskr. Berlin, Julius Springer, 1932.

6. Conners, C. A., EckhardT, R. E. and Johnson, L. V.-Arch. Ophthal. Vol. XXIX, p. 958, 1943.

7. Johnson, L. V. and EckHARDT, R. E.-Arch. Ophthal., Vol. XXIII, p. 899, 1940.

8. FisH, W. M.-Amer. Jl. Ophthal., Vol. XXVII, p. 354, 1944.

9. Wise, G.-Amer. Jl. Ophthal,, Vol. XXVI, p. 291, 1943.

10. Sulzberger, M. B. and Copt, E. P.-Jl. Lab. Clin. Med., Vol. XXVI, p. $1403,1941$.

11. DukE-Elder.-Text-book of Ophthalmology, Vol. II, p. 1726, Ed. London. H. Kimpton. 1943.

12. ThIеL, R.-Klin. Monatsbl. f. Augenheilk., Vol. CVIII, p. 394, 1939.

13. LieBenBerg, H. S.-A mer. Jl. Ophthal., Vol. XXVI, p. 307, 1943.

14. Elschnig, A.-Arch.f. Ophthal., Vol. LXXVI, p. 509, 1919.

15. Woods, A. L.-Arch. of Ophthal., Vol. XVII, p. 283, 1917.

16. VeRhoEFF, F. H. and LemorNe, A.. N. - International Congress of Ophthalmology, Washington, 1922. William F. Pile Co, Philadelphia, 1922.

17. FriedenWALD, J. S.-Amer. Jl. Ophthal., Vol. XVII, p. 1008, 1934.

18. ApPelbaum, A.-Arch. of Ophthal., Vol. XXIV, p. 820, 1940.

19. Goodman, E. I.--Arch. of Ophthal., Vol. XIV, p. 90, 1935.

\section{SOME OBSERVATIONS ON THE SYMPTOMATOLOGY AND DIAGNOSIS OF CASES OF PROPTOSIS *

\author{
BY \\ Professor HaNDOUSA BEY \\ CAIRO
}

I HAVE had to examine and treat a large number of cases of proptosis, most of them referred to me by the various ophthalmic centres in Egypt. Study of the records of these cases reveals some valuable points worthy of publication.

The types of cases that I have come across, fall into the following categories:-

1.-Cases of Extra-Orbital Causation.

2.-Cases of Intra-Orbital Causation.

3.-Cases due to disease of the bony wall of the orbital cavity.

* Received for publication, October 18, 1946. 